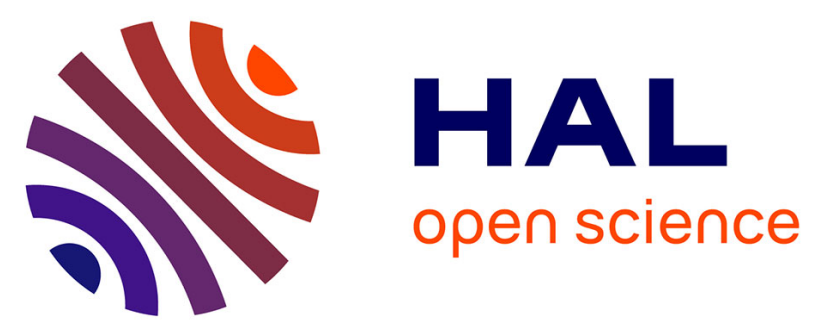

\title{
Influence of the magnetic field sweeping rate on magnetic transitions in synthetic ferrimagnets with perpendicular anisotropy
}

R. B Morgunov, E I Kunitsyna, A. D Talantsev, O. V Koplak, T. Fache, Yuan Lu, S. Mangin

\section{- To cite this version:}

R. B Morgunov, E I Kunitsyna, A. D Talantsev, O. V Koplak, T. Fache, et al.. Influence of the magnetic field sweeping rate on magnetic transitions in synthetic ferrimagnets with perpendicular anisotropy. Applied Physics Letters, 2019, 114 (22), pp.222402. 10.1063/1.5096951 . hal-02999627v2

\section{HAL Id: hal-02999627 \\ https://hal.science/hal-02999627v2}

Submitted on 1 Dec 2019

HAL is a multi-disciplinary open access archive for the deposit and dissemination of scientific research documents, whether they are published or not. The documents may come from teaching and research institutions in France or abroad, or from public or private research centers.
L'archive ouverte pluridisciplinaire HAL, est destinée au dépôt et à la diffusion de documents scientifiques de niveau recherche, publiés ou non, émanant des établissements d'enseignement et de recherche français ou étrangers, des laboratoires publics ou privés. 


\section{Influence of the magnetic field sweeping rate on magnetic transitions in synthetic ferrimagnets with perpendicular anisotropy}

Cite as: Appl. Phys. Lett. 114, 222402 (2019); https://doi.org/10.1063/1.5096951

Submitted: 21 March 2019 . Accepted: 16 May 2019 . Published Online: 04 June 2019

R. B. Morgunov (D) E. I. Kunitsyna (D), A. D. Talantsev (D), O. V. Koplak, T. Fache, Y. Lu (D), and S. Mangin
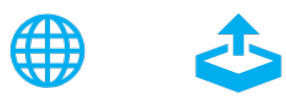

\section{ARTICLES YOU MAY BE INTERESTED IN}

Cross-shaped nanostructures for the study of spin to charge inter-conversion using spin-orbit coupling in non-magnetic materials

Applied Physics Letters 114, 222401 (2019); https://doi.org/10.1063/1.5078957

Magneto-dynamic properties of complex oxide-La0.7 $\mathrm{SrO}_{3} \mathrm{MnO}_{3} / \mathrm{SrTiO}_{3}$-heterostructure interface

Applied Physics Letters 114, 222403 (2019); https://doi.org/10.1063/1.5093324

Enhanced ferroelectricity in epitaxial $\mathrm{Hf}_{0.5} \mathrm{Zr} 0.5 \mathrm{O}_{2}$ thin films integrated with $\mathrm{Si}(001)$ using $\mathrm{SrTiO}_{3}$ templates

Applied Physics Letters 114, 222901 (2019); https://doi.org/10.1063/1.5096002

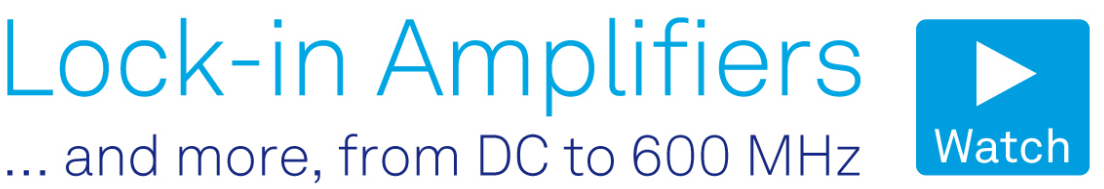




\title{
Influence of the magnetic field sweeping rate on magnetic transitions in synthetic ferrimagnets with perpendicular anisotropy
}

Cite as: Appl. Phys. Lett. 114, 222402 (2019); doi: 10.1063/1.5096951

Submitted: 21 March 2019 • Accepted: 16 May 2019 .

Published Online: 4 June 2019

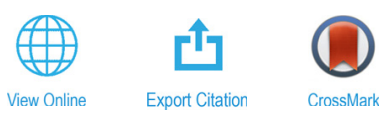

R. B. Morgunov, ${ }^{1,2, a)}$ (D) E. I. Kunitsyna, ${ }^{1,2}$ (D) A. D. Talantsev, ${ }^{1,2,3}$ (D) O. V. Koplak, ${ }^{1,2}$ T. Fache, $^{4}$ Y. Lu, ${ }^{4}$ (D) and S. Mangin ${ }^{4}$

\author{
AFFILIATIONS \\ ${ }^{7}$ Institute of Problems of Chemical Physics, 142432 Chernogolovka, Russia \\ ${ }^{2}$ Tambov State Technical University, 392000 Tambov, Russia \\ ${ }^{3}$ Department of Emerging Materials Science, DGIST, Daegu 42988, South Korea \\ ${ }^{4}$ Institut Jean Lamour, UMR 7198 CNRS, Université de Lorraine, Nancy 54601, France
}

a)E-mail: morgunov2005@yandex.ru

\begin{abstract}
The evolution of the switching field between stable states in $\mathrm{MgO} / \mathrm{CoFeB} / \mathrm{Ta} / \mathrm{CoFeB} / \mathrm{MgO} / \mathrm{GaAs}$ and $\mathrm{Pt} / \mathrm{Co} / \mathrm{Ir} / \mathrm{Co} / \mathrm{Pt} / \mathrm{Ta} / \mathrm{SiO}{ }_{2}$ synthetic ferrimagnets with perpendicular anisotropy as a function of the magnetic field sweeping rate (MFSR) of $0.1-10^{4} \mathrm{Oe} / \mathrm{s}$ has been studied. The most significant effect of the MFSR is an inversion of the interstate transition sequence. In the $\mathrm{MgO} / \mathrm{CoFeB} / \mathrm{Ta} / \mathrm{CoFeB} / \mathrm{MgO} / \mathrm{GaAs}$ heterostructure, the increase in MFSR switches the dominant mechanism of magnetization reversal from propagation of domain walls to a nucleation of reversed magnetization areas. In $\mathrm{Pt} / \mathrm{Co} / \mathrm{Ir} / \mathrm{Co} / \mathrm{Pt} / \mathrm{Ta} / \mathrm{SiO}_{2}$, the $\mathrm{MFSR}$ affects the final domain state of the transition, starting from the initial antiparallel configuration of the synthetic ferrimagnet.
\end{abstract}

Published under license by AIP Publishing. https://doi.org/10.1063/1.5096951

In the last ten years, magnetic relaxation of multilayered ferromagnetic heterostructures has attracted a lot of interest due to its importance in the understanding of the magnetization switching dynamics. $^{1-3}$ Magnetic relaxations in the $\mathrm{MgO} / \mathrm{CoFeB} / \mathrm{Ta} / \mathrm{CoFeB} /$ $\mathrm{MgO} / \mathrm{GaAs}^{4}$ and $\mathrm{Pt} / \mathrm{Co} / \mathrm{Ir} / \mathrm{Co} / \mathrm{Pt} / \mathrm{Ta} / \mathrm{Si}^{5}$ synthetic ferrimagnets with perpendicular magnetic anisotropy (PMA) have already been studied. The time scales of these relaxations range from a few seconds to several hours. The duration of the magnetization reversal depends on the potency (expectation time) of the domain wall depinning and efficacy (kinetics) of nucleation of the reversed magnetization phase. These two mechanisms of the magnetic relaxation were combined in the FatuzzoLabrune model, ${ }^{6,7}$ describing the dynamics of magnetic relaxation in single ferromagnetic thin films, fully understood in the past few years. Magnetic relaxation contributes to the shape of the hysteresis loop as well. ${ }^{8}$ The shape of the hysteresis loop and switching fields between the parallel and antiparallel states of synthetic antiferromagnets are strongly affected by the magnetic field sweeping rate (MFSR), when the duration of the loop recording is comparable to the magnetic relaxation time. The latter depends on the lateral size of the heterostructure. If the lateral size is of a few nanometers and comparable with the thicknesses of the layers, the typical switching time $\sim 1 \mathrm{~ns}$ is shorter by many orders of magnitude than the duration of a hysteresis loop recording by a Kerr microscope ( $\sim 1$ s), a vibration sample magnetometer ( $\sim 1 \mathrm{~min})$, and a superconducting quantum interference device (SQUID) magnetometer $(\sim 1 \mathrm{~h})$. Thus, the values of magnetization reversal parameters in nanosized heterostructures as nanopillars and nanodots are independent of the measurement technique used. In contrast, heterostructures of a large lateral size $(5 \mathrm{~mm} \times 5 \mathrm{~mm}$ or larger) manifest long-lasting magnetic relaxation dynamics $\left(\sim 10^{3}-10^{4} \mathrm{~s}\right)$, limited by the domain propagation time throughout the sample area, ${ }^{9,10}$ and the duration of magnetization reversal can become comparable to the magnetic hysteresis loop recording time. In that case, the observed value of the interstate transition field is expected to be strongly dependent on MFSR.

In general, synthetic antiferromagnets with two ferromagnetic layers of different thicknesses show four stable states, $\mathrm{P}^{+}, \mathrm{AP}^{+}, \mathrm{AP}^{-}$, and $\mathrm{P}^{-}$[Fig. 1(a)], corresponding to parallel and antiparallel mutual orientations of magnetizations in top and bottom layers ( $\mathrm{P}$ and $\mathrm{AP}$, respectively) and the positive or negative sign of the net magnetic moment value (indicated by a "+" or “-” sign). The magnetic field range, at which the interstate transition occurs, is dependent on the balance between the energy terms of the synthetic antiferromagnet under an applied magnetic field: magnetic energy of the layers, 

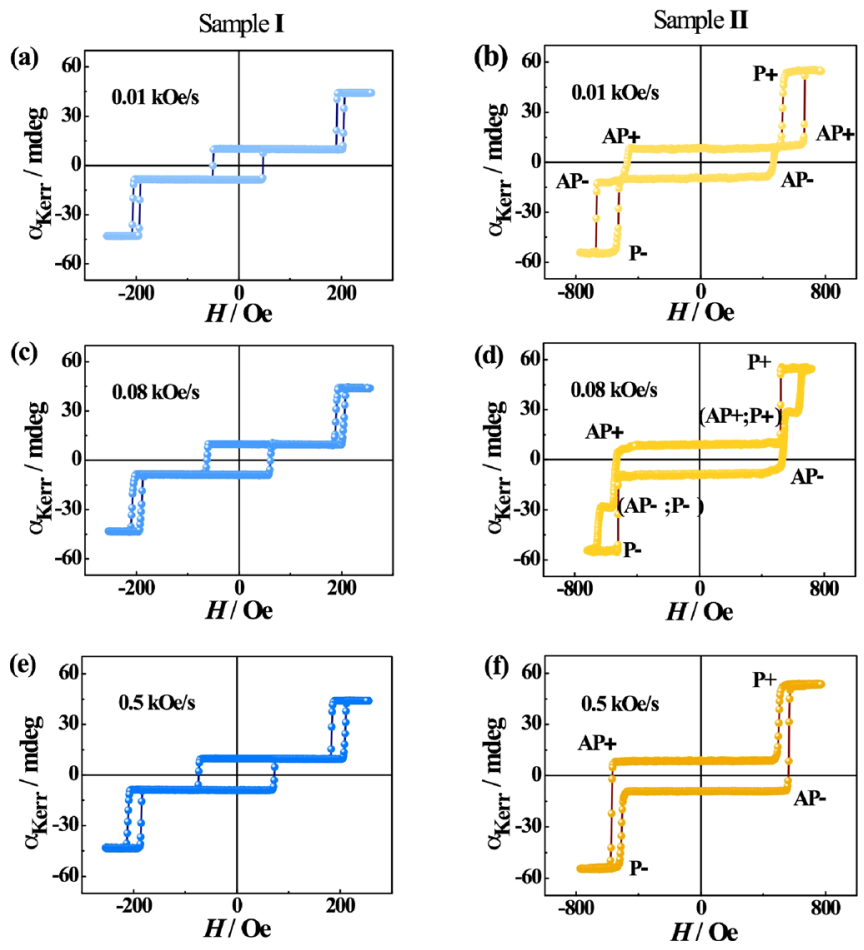

FIG. 1. MOKE hysteresis loops of sample I [(a), (c), and (e)] and sample II [(b), (d), and (f)], recorded at $T=300 \mathrm{~K}$ at $0.01 \mathrm{kOe} / \mathrm{s}, 0.08 \mathrm{kOe} / \mathrm{s}$, and $0.5 \mathrm{kOe} / \mathrm{s}$ magnetic field sweep rates.

anisotropy energies, domain unpinning barriers, and interlayer RKKY coupling. In this work, the thicknesses of the Co ferromagnetic layers and the $\mathrm{Ir}$ spacer layer in the $\mathrm{Pt} / \mathrm{Co} / \mathrm{Ir} / \mathrm{Co} / \mathrm{Pt} / \mathrm{Ta} / \mathrm{SiO}_{2}$ structure were selected in a way that the $\mathrm{AP}^{+}$to $\mathrm{AP}^{-}$transition (synchronous reversals of the top and bottom Co layers) and the $\mathrm{AP}^{+}$to $\mathrm{P}$-transition (reversal of the bottom Co layer only) proceed in overlapping field ranges. The $\mathrm{MgO} / \mathrm{CoFeB} / \mathrm{Ta} / \mathrm{CoFeB} / \mathrm{MgO} / \mathrm{GaAs}$ structure was selected as a reference sample, where the transition fields do not overlap. This work is aimed at the experimental analysis of the MSFR effect on transition fields and the transition sequence for the "overlapping" case and comparison of this effect with the conventional one in synthetic antiferromagnets without an overlap of transition fields.

The $\mathrm{MgO}(2.5 \mathrm{~nm}) / \mathrm{CoFeB}(1.1 \mathrm{~nm}) / \mathrm{Ta}(0.75 \mathrm{~nm}) / \mathrm{CoFeB}(0.8 \mathrm{~nm}) /$ $\mathrm{MgO}(2.5 \mathrm{~nm}) / \mathrm{Ta}$ (5 nm) [Fig. S1(a)] double ferromagnetic layer system was deposited on the undoped GaAs (001) substrate by magnetron sputtering. The Energy Dispersive X-Ray Spectroscopy (EDX) chemical analysis of the layers and TEM images of the cross section of this heterostructure are presented in Ref. 11. Continuous and homogeneous $3 \times 3 \mathrm{~mm}^{2}$ plate-shaped layer structures with perpendicular anisotropy were identified.

The multilayered structure $\mathrm{Pt}(3 \mathrm{~nm}) / \mathrm{Co}(1.0 \mathrm{~nm}) / \mathrm{Ir}(1.5 \mathrm{~nm}) / \mathrm{Co}$ $(1.5 \mathrm{~nm}) / \mathrm{Pt}(3 \mathrm{~nm}) / \mathrm{Ta}(3 \mathrm{~nm}) / \mathrm{SiO}_{2}$ [Fig. S1(b)] was prepared by deposition on the undoped $\mathrm{Si} / \mathrm{SiO}_{2}$ substrate of size $0.4 \times 4 \times 5 \mathrm{~mm}^{3}$ by magnetron sputtering in $10^{-8}$ Torr vacuum. The iridium spacer allows one to predict the oscillating RKKY exchange interaction as a function of its thickness. ${ }^{12}$

The recording of dynamic hysteresis loops and domain observation at room temperature $(300 \mathrm{~K})$ were performed using a Durham
Magneto-optics NanoMOKE3 Magneto Optical Kerr Effect (MOKE) measurement system, in the polar MOKE measurement configuration. The domain imaging was performed at a $2 \mathrm{Oe} / \mathrm{s}$ field sweep rate with an image scanning time of $0.5 \mathrm{~s}$ per frame, which was $10^{2}-10^{4}$ times less than the time of domain wall propagation through the microscope observation area.

Microwave nonresonant absorption was recorded using a Bruker ESP $300 \mathrm{X}$-band ESR spectrometer (microwave frequency was $f_{0}$ $=9.447 \mathrm{GHz}$, microwave power $6.3 \mathrm{~mW}$, modulation frequency $100 \mathrm{kHz}$, modulation amplitude $10 \mathrm{Oe}$, and quality factor $\mathrm{Q} \sim 4000$ ). If the resistance of the sample is lower than the resistance of the microwave circuit $\left(R_{\mathrm{C}} \sim 190 \Omega\right)$, absorbed microwave power $P$ is proportional to sample resistance $\rho$ and depends on magnetic field $P \sim \rho(H) .{ }^{13-15}$ Thus, in our experiments, the derivation of absorbed microwave power measured by the modulation method was directly proportional to the derivation of specific resistance $\rho$ of the sample $d P / d H \sim d(\rho(H)) / d H$. Magnetic hysteresis loops were recorded using an MPMS 5XL Quantum Design superconducting quantum interference device (SQUID) magnetometer. The sweeping rate in this method was the slowest in comparison with the two methods mentioned above.

The magnetic hysteresis loops recorded by the MOKE technique at three different MFSR values in the CoFeB bilayer (sample I) at $300 \mathrm{~K}$ are presented in Figs. 1(a), 1(c), and 1(e). Parallel and antiparallel alignments of the magnetizations of the bottom layer ( $0.8 \mathrm{~nm}$ thickness) and top layer $(1.1 \mathrm{~nm})$ thickness result in four stable magnetic states $\mathrm{P}^{+}, \mathrm{P}^{-}$, $\mathrm{AP}^{+}$, and $\mathrm{AP}^{-}$. Transitions between the stable states result in three minor hysteresis loops: the central one and the two side ones, shifted from zero field by $\sim 200$ Oe due to the negative exchange coupling between both CoFeB layers. ${ }^{16}$ The transition from the $\mathrm{AP}^{+}$to $\mathrm{AP}^{-}$state starts by nucleation of $\mathrm{AP}^{-}$domains and their growth by propagation of $\mathrm{AP}^{+} / \mathrm{AP}^{-}$domain walls. A series of the microimages of one of the magnetic nuclei at MFSR $=2 \mathrm{Oe} / \mathrm{s}$ are presented in Fig. 2(a). The switching fields of the central (Fig. S2) and side (Fig. S3) hysteresis loops depend on the MFSR during loop recording. With an increase in the MFSR from $0.1 \mathrm{Oe} / \mathrm{s}$ (typical for the SQUID magnetometer) to $10 \mathrm{kOe} / \mathrm{s}$ (typical for the MOKE technique), the $\mathrm{AP}^{+} \rightarrow \mathrm{AP}^{-}$transition field grows 3 times, from 20 Oe to 60 Oe [Fig. 3(a)]. This growth of the switching field with MSFR can be described in terms of energy balance for the synthetic antiferromagnet.

In a single thin film, the dependence of the switching critical field, $H_{\mathrm{C}}$, on the MFSR value $\mathrm{d} H / \mathrm{d} t$ typically follows the logarithmic law: ${ }^{17,18}$

$$
H_{C}(R)=H_{\mathrm{f}} \ln (R)+\text { const, }
$$

where $H_{\mathrm{f}}$ is the fluctuation field and $R=\mathrm{d} H / \mathrm{d} t$ is MFSR.

To extend this equation for synthetic antiferromagnets, the interlayer coupling energy, magnetic energies of two layers, and anisotropy energies should be taken into account.

For $\mathrm{AP}^{+} \rightarrow \mathrm{AP}^{-}$transition,

$$
\begin{gathered}
H_{C}=H_{f} \ln \left(\frac{R}{R_{0}}\right)+\frac{K_{A}}{2 M_{S}} \frac{h_{2}+h_{1}}{h_{2}-h_{1}}, \\
H_{f}=\frac{k T}{2 M_{S} V_{A}} \frac{h_{2}+h_{1}}{h_{2}-h_{1}}, \\
R_{0}=\frac{f_{0} k T}{M_{S} V_{A}} \frac{h_{2}+h_{1}}{h_{2}-h_{1}},
\end{gathered}
$$


(a) Sample I:
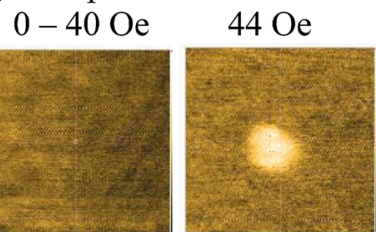

(b) Sample II:

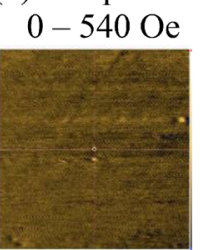

48 Oe

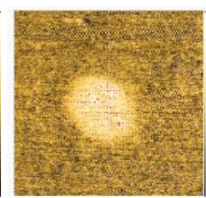

550 Oe

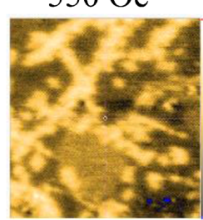

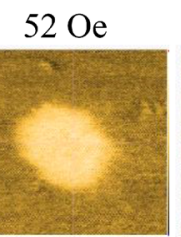

555 Oe

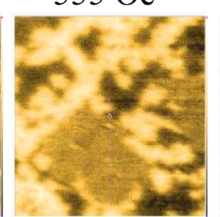

$56 \mathrm{Oe}$

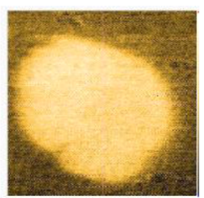

560 Oe

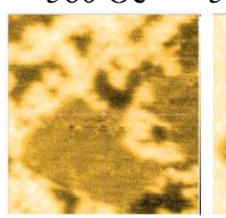

$60-200$ Oe

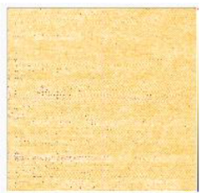

$565-7500$

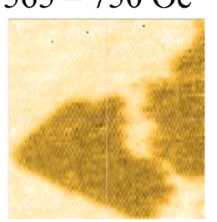

FIG. 2. MOKE images of domains, corresponding to the $\mathrm{AP}^{+} \rightarrow \mathrm{AP}^{-}$transition field range, in sample I (MgO/CoFeB/Ta/ $\mathrm{CoFeB} / \mathrm{MgO})$ (a) and sample II (Pt/Co/Ta/ $\mathrm{Co} / \mathrm{Pt}$ ) (b), recorded at $T=300 \mathrm{~K}$ at a 20 e/s magnetic field sweep rate. where $K_{\mathrm{A}}$ is the anisotropy constant, $V_{\mathrm{A}}$ is the activation volume, $M_{\mathrm{S}}$ is the saturation magnetization $\left(K_{\mathrm{A}}\right.$ and $M_{\mathrm{S}}$ values were supposed to be similar for top and bottom layers of synthetic antiferromagnet), $h_{1}$ and $h_{2}$ are the thicknesses of top and bottom layers, respectively, and $f_{0}$ is the attempt frequency (Arrhenius factor) of domain nucleation. The interlayer coupling energy is not present in Eqs. (2)-(4), as the $\mathrm{AP}^{+} \rightarrow \mathrm{AP}^{-}$transition proceeds between two antiparallel states and, therefore, does not change the sign of interlayer coupling. However, the switching field depends on interlayer coupling energy
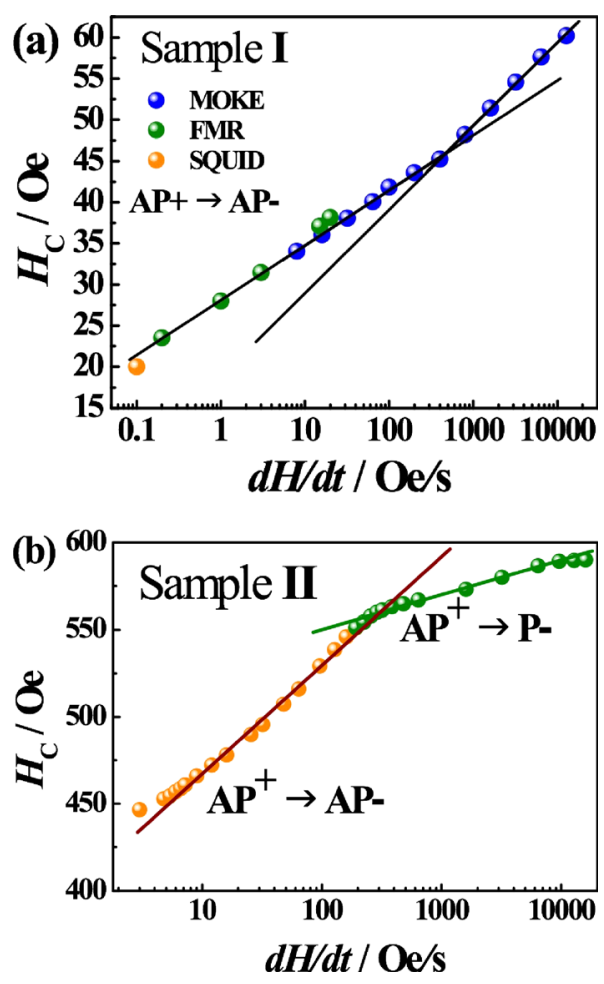

FIG. 3. The dependence of the transition field on the magnetic field sweep rate (semilogarithmic scale), for sample I (MgO/CoFeB/Ta/CoFeB/MgO) (a) and sample II $(\mathrm{Pt} / \mathrm{Co} / \mathrm{lr} / \mathrm{Co} / \mathrm{Pt})(\mathrm{b})$. Solid lines are linear approximations. for $\mathrm{P}^{+} \rightarrow \mathrm{AP}^{+}$and $\mathrm{AP}^{-} \rightarrow \mathrm{P}^{-}$transitions, when the mutual orientation of magnetizations in top and bottom layers changes from collinear to opposite and vice versa (See Appendix I in the supplementary material).

The derivation of these equations and the formulas for other transitions in the synthetic antiferromagnet are given in Appendix I supplementary material. Equation (3) for a fluctuation field in a bilayer system is similar to the one for single layer thin films, ${ }^{19}$

$$
H_{\mathrm{f}}=k T /\left(2 V_{\mathrm{A}} M_{\mathrm{S}}\right),
$$

with an exception of the $\left(h_{2}+h_{1}\right) /\left(h_{2}-h_{1}\right)$ term. The physical meaning of this term is that for the same temperature and activation volume, the fluctuation field in a bilayer is much larger than that in a single layer thin film. The $H_{\mathrm{f}}$ value depends on the mechanism of the domain wall pinning and is sensitive to the "strong" or "weak" pinning cases. $^{20,21}$ Thus, the $H_{\mathrm{f}}$ parameter characterizes the thermal stability of the material, being dependent on the ratio between the energy of the reversal magnetization phase nucleation and the energy of the domain wall pinning by structural defects. Since the thicknesses of the magnetic layers in our samples $(0.8 \mathrm{~nm}$ and $1.1 \mathrm{~nm})$ are of a few lattice parameters $(0.4 \mathrm{~nm})$, any defect of crystalline structure corresponds to a local change of the film thickness by $20 \%-30 \%$. For that reason, the fluctuation field $H_{\mathrm{f}}$ is comparable to the coercive field $H_{\mathrm{C}}$ (Table 1 in the supplementary material) and can be changed by few times in contrast to the bulk sample or thick $(>10 \mathrm{~nm})$ films, where the change of the MFSR by few orders of value causes a change in $H_{\mathrm{f}}$ by few percent. Thus, in the thin films, the results of measurements of the fluctuation field strongly depend on the method of measurement [in SQUID MFSR $=0.1 \mathrm{Oe} / \mathrm{s}$, in Ferromagnetic resonance (FMR) MFSR $=0.2-20 \mathrm{Oe} / \mathrm{s}$ (Fig. S4), and in MOKE microscope MFSR $=10 \mathrm{kOe} / \mathrm{s}]$.

The $H_{\mathrm{C}}(\ln R)$ dependence has a kink at $R \sim 500$ Oe/s [Fig. 3(a)]. This kink cannot be explained by the change of the sign of the derivative of domain wall velocity in the magnetic field close to the Walker limit. ${ }^{22,23}$ A more realistic explanation of the kink can be proposed on the basis of consideration of the competition between two ways of transitions from the $\mathrm{AP}^{+}$to $\mathrm{AP}^{-}$state: by nucleation of the magnetization reversal phase and propagation of the domain walls. In this mode, the number of nuclei becomes dependent on the external magnetic field before full magnetization reversal of the film is realized by the growth of nuclei. Thus, the activation volume and fluctuation field 
in high MFSR modes are controlled by both the nucleation field and the depinning field (see Fig. S5 in the supplementary material).

An evolution of the magnetic hysteresis loop with MFSR for the $\mathrm{Pt} / \mathrm{Co} / \mathrm{Ir} / \mathrm{Co} / \mathrm{Pt} / \mathrm{Ta} / \mathrm{SiO}_{2}$ structure (sample II) is presented in Figs. 1(b), 1(d), and 1(f). In addition to the shift of transition fields, an increase in MFSR results in the inversion of interstate transition sequence. At slow field sweep rates (below $0.01 \mathrm{kOe} / \mathrm{s}$ ), the switching from the $\mathrm{AP}^{+}$state to the $\mathrm{P}^{-}$state proceeds via the $\mathrm{AP}^{-}$state as two separate transitions: (i) $\mathrm{AP}^{+} \rightarrow \mathrm{AP}^{-}$- synchronous propagation of the $\mathrm{AP}^{+} / \mathrm{AP}^{-}$domain wall in top and bottom Co layers in the 470-520 Oe field range and (ii) $\mathrm{AP}^{-} \rightarrow \mathrm{P}^{-}$- nucleation of domains in the top Co layer, in the 720-750 Oe field range. With an increase in MFSR, it is expected that the $\mathrm{AP}^{+} \rightarrow \mathrm{AP}^{-}$transition field will increase, until it reaches the $\mathrm{AP}^{-}$ $\rightarrow \mathrm{P}^{-}$transition field ( $\left.\sim 750 \mathrm{Oe}\right)$ and the $\mathrm{AP}^{-}$state is no more dwelled. However, a different process is experimentally observed. The $\mathrm{AP}^{+}$ $\rightarrow \mathrm{AP}^{-}$transition field does not reach the $\mathrm{AP}^{-} \rightarrow \mathrm{P}^{-}$transition field. Instead, at $\sim 550$ Oe, a domain nucleation in the bottom layer, resulting in the direct transition from the $\mathrm{AP}^{+}$to $\mathrm{P}^{-}$state, starts to proceed together with the $\mathrm{AP}^{+} \rightarrow \mathrm{AP}^{-}$one. When the MFSR is in a range of $0.02 \mathrm{kOe} / \mathrm{s}-0.45 \mathrm{kOe} / \mathrm{s}$, these two processes occur together and competitively, leading to a multidomain stable magnetic state $\left(\mathrm{AP}^{-} ; \mathrm{P}^{-}\right)$, lying in between $\mathrm{AP}^{-}$and $\mathrm{P}^{-}$[Fig. 1(d)].

A series of MOKE images recorded in intermediate MFSR is shown in Fig. 2(b). Three types of areas can be distinguished in these images: Dark areas correspond to the $\mathrm{AP}^{+}$state, the areas of the intermediate brightness correspond to the $\mathrm{AP}^{-}$state, and light areas correspond to the $\mathrm{P}^{-}$state. When this transition is completed [Fig. 2(b), last image], domains of two states $\mathrm{AP}^{-}$and $\mathrm{P}^{-}$coexist and this $\left(\mathrm{AP}^{-}\right.$; $\mathrm{P}^{-}$) state is stable in the $550 \mathrm{Oe}-750$ Oe field range. The proportion between the areas, occupied by $\mathrm{AP}^{-}$and $\mathrm{P}^{-}$domains, was found to be dependent on MFSR (Fig. S6, supplementary material). When the field reaches $750 \mathrm{Oe}$, those areas, which are in the $\mathrm{AP}^{-}$state, switch to the $\mathrm{P}^{-}$state, until all the areas of the film are switched to the $\mathrm{P}^{-}$state.

At high MFSR values $(>0.5 \mathrm{kOe} / \mathrm{s})$, the transition in 750 Oe disappears [Fig. 1(f)] and the reversal from the $\mathrm{AP}^{+}$to $\mathrm{P}^{-}$state becomes fully completed at a lower magnetic field, $\sim 600 \mathrm{Oe}$, corresponding to the nucleation of magnetic domains in the bottom Co layer. It means that at high MFSR, the reversal from $\mathrm{AP}^{+}$to $\mathrm{P}^{-}$is localized in the bottom Co layer only. No formation of domain nuclei in the top Co layer occurs at high MFSR. In contrast, at low MFSR values, due to the $\mathrm{AP}^{+}$ to $\mathrm{AP}^{-}$preceding process, the domain nucleation occurs in the top Co layer and does not happen in the bottom Co layer. Thus, an increase in MFSR results in relocalization of the domain nucleation process from the top to the bottom Co layer, by suppression of propagation of $\mathrm{AP}^{+} / \mathrm{AP}^{-}$domain walls. The evidence of this relocalization are (i) the appearance of the direct $\mathrm{AP}^{+} \rightarrow \mathrm{P}^{-}$process, corresponding to domain nucleation in the bottom Co layer, at intermediate MFSR, and (ii) the disappearance of transition at $750 \mathrm{Oe}$, corresponding to domain nucleation in the top Co layer, at high MFSR.

Dynamics of the $\mathrm{AP}^{-}$and $\mathrm{P}^{-}$domain nucleation and propagation is summarized in Fig. S5(b) of the supplementary material for three ranges of the MFSR: Slow mode $(<0.01 \mathrm{kOe} / \mathrm{s})$, intermediate mode $(0.02 \mathrm{kOe} / \mathrm{s}-0.45 \mathrm{kOe} / \mathrm{s})$, and fast mode $(>0.5 \mathrm{kOe} / \mathrm{s})$. For the transitions, starting from the $\mathrm{AP}^{+}$state, the dependences of switching fields on MFSR are presented in Fig. 3(b). The fluctuation field and the activation volume, extracted by linear approximations of the straight parts of these dependences by formula (1), are presented in Table 1 of the supplementary material. These values are close to the ones obtained in $\mathrm{SmCo}_{5} / \mathrm{Fe} / \mathrm{SmCo}_{5}$ trilayers ${ }^{3}$ and $\mathrm{Co} / \mathrm{Pt}$ multilayer structures. ${ }^{24}$ The $H_{\mathrm{C}}(d H / d t)$ dependences abruptly change the slope at the critical $d H / d t$ value in the CoFeB sample, as well as in the Pt/Co sample. However, the origins of these features are different in $\mathrm{Pt} / \mathrm{Co}$ and $\mathrm{CoFeB}$ synthetic ferrimagnets. In the $\mathrm{CoFeB}$ based heterostructures, the slope increases due to the progressive involvement of the domain nucleation process with the increase in MFSR. In contrast to $\mathrm{CoFeB}$ synthetic ferrimagnets, in the $\mathrm{Pt} / \mathrm{Co}$ samples, the slope of $H_{\mathrm{C}}(d H / d t)$ decreases, and it is caused by migration of the final magnetization state. At low MFSR values, the $\mathrm{AP}^{+} \rightarrow \mathrm{AP}^{-}$transition takes place, while at high MFSR values, the $\mathrm{AP}^{+} \rightarrow \mathrm{P}^{-}$transition occurs. Different slopes of the dependence of switching field $H_{\mathrm{C}}$ on the sweeping rate $R$ $=d H / d t$ in logarithmic coordinates $\left[H_{\mathrm{C}} ; \ln (R)\right][$ Fig. $3(\mathrm{~b})]$ indicate the difference between the fluctuation fields and activation volumes corresponding to the $\mathrm{AP}^{+} \rightarrow \mathrm{AP}^{-}$and $\mathrm{AP}^{+} \rightarrow \mathrm{P}^{-}$transitions most probably due to different heights of the activation barriers of these transitions. The $\mathrm{AP}^{+} \rightarrow \mathrm{P}^{-}$transition corresponds to the magnetization reversal of just one of the ferromagnetic layers. Thus, the realization of the $\mathrm{AP}^{+} \rightarrow \mathrm{P}^{-}$transition requires overcoming the barrier $E_{1}$ corresponding to the single layer, while the $\mathrm{AP}^{+} \rightarrow \mathrm{AP}^{-}$transition corresponds to the magnetization reversal of both the layers requiring to overcome the sum of the activation energies $E_{1}+E_{2}$ of both the ferromagnetic layers.

In multilayered synthetic ferrimagnets $\mathrm{Pt} / \mathrm{Co} / \mathrm{Ir} / \mathrm{Co} / \mathrm{Pt}$ and $\mathrm{CoFeB} / \mathrm{Ta} / \mathrm{CoFeB}$ with perpendicular anisotropy, the magnetic field sweeping rate dependence of the switching field between different stable states has been investigated. The increase in the sweeping rate on 5 orders of magnitude from $0.1 \mathrm{Oe} / \mathrm{s}$ up to $10 \mathrm{kOe} / \mathrm{s}$ changes the $\mathrm{AP}^{+}$ $\rightarrow \mathrm{AP}^{-}$switching field in $\mathrm{CoFeB} / \mathrm{Ta} / \mathrm{CoFeB}$ by a factor of 3 (from 20 Oe to $60 \mathrm{Oe}$ ). More interestingly, in a $\mathrm{Pt} / \mathrm{Co} / \mathrm{Ir} / \mathrm{Co} / \mathrm{Pt}$ heterostructure, the sequence of the transitions between stable states can be changed by controlling the sweeping rate of the magnetic field.

Two mechanisms could be proposed to explain those behaviors. In $\mathrm{CoFeB} / \mathrm{Ta} / \mathrm{CoFeB}$ by modifying the sweeping rate, the magnetization reversal changes from domain wall propagation to nucleation, dominating at a high sweeping rate. In the $\mathrm{Pt} / \mathrm{Co} / \mathrm{Ir} / \mathrm{Co} / \mathrm{Pt}$ structure, an increase in the magnetic sweeping rate changes the type of switching from $\mathrm{AP}^{+} \rightarrow \mathrm{AP}^{-}$and $\mathrm{AP}^{-} \rightarrow \mathrm{P}^{-}$transitions. Simultaneous contributions of the $\mathrm{AP}^{+} \rightarrow \mathrm{AP}^{-}$and $\mathrm{AP}^{+} \rightarrow \mathrm{P}^{-}$transitions to magnetization reversal result in a stable multidomain state of the heterostructure, with coexisting domains of $\mathrm{AP}^{-}$and $\mathrm{P}^{-}$states.

An increase in MFSR results in the relocalization of the domain nucleation process from the top to the bottom Co layer, by suppression of the propagation of $\mathrm{AP}^{+} / \mathrm{AP}^{-}$domain walls. The evidences of this relocalization are (i) the appearance of a direct $\mathrm{AP}^{+} \rightarrow \mathrm{P}^{-}$process, corresponding to domain nucleation in the bottom Co layer, at intermediate MFSR, and (ii) the disappearance of transition at $750 \mathrm{Oe}$, corresponding to domains nucleation in the top Co layer, at high MFSR.

See the supplementary material for the structure of two samples, complete ESR and MOKE data of the samples, a sketch of the nucleation for different MFSR, and activation volumes of the transitions.

R.M., E.K., A.T., and O.K. were supported by the Ministry of Education and Science of Russian Federation (Grant No. 
3.1992.2017/4.6). A.T. was supported by the R\&D program of MOTIE/KEIT (No. 10064089, Development of low power current sensor and module for automotive with $0.1 \%$ precision) and the DGIST R\&D Program of the Ministry of Science, ICT and Future Planning (No. 19-BT-02). S.M., T.F., and Y.L. were supported by the ANR-NSF Project, No. ANR-13-IS04-0008-01, COMAG, No. ANR-15-CE24-0009 UMAMI, by the ANR-Labcom Project LSTNM, by the Institut Carnot ICEEL for the project "Opticswitch" and Matelas, and by the French PIA project "Lorraine Université d'Excellence," Reference No. ANR-15IDEX-04-LUE. Experiments were performed using equipment from the TUBE Davm, funded by FEDER (EU), ANR, Région Grand Est and Métropole Grand Nancy.

\section{REFERENCES}

'J. E. Davies, O. Hellwig, E. E. Fullerton, and K. Liu, Phys. Rev. B 77, 014421 (2008).

${ }^{2}$ H. Liu, D. Bedau, J. Z. Sun, S. Mangin, E. E. Fullerton, J. A. Katine, and A. D. Kent, J. Magn. Magn. Mater. 358-359, 233 (2014).

${ }^{3}$ S. J. Collocott and V. Neu, J. Phys. D: Appl. Phys. 45, 035002 (2012).

${ }^{4}$ R. Morgunov, Y. Lu, M. Lavanant, T. Fache, X. Deveaux, S. Migot, O. Koplak, A. Talantsev, and S. Mangin, Phys. Rev. B 96, 054421 (2017).

${ }^{5}$ A. Talantsev, Y. Lu, T. Fache, M. Lavanant, A. Hamadeh, A. Aristov, O. Koplak, R. Morgunov, and S. Mangin, J. Phys.: Condens. Matter 30, 135804 (2018).

${ }^{6}$ M. Labrune, S. Andrieu, F. Rio, and P. Bernstein, "Time dependence of the magnetizaton process of Re-TM alloys," J. Magn. Magn. Mater. 80, 211 (1989).

${ }^{7}$ E. Fatuzzo, "Theoretical considerations on the switching transient in ferroelectrics,” Phys. Rev. 127, 1999 (1962).
${ }^{8}$ X. Xu, T. Kihara, M. Tokunaga, A. Matsuo, W. Ito, R. Y. Umetsu, K. Kindo, and R. Kainuma, Appl. Phys. Lett. 103, 122406 (2013).

${ }^{9}$ R. B. Morgunov, G. L. L'vova, A. D. Talantsev, O. V. Koplak, T. Fache, and S. Mangin, J. Magn. Magn. Mater. 459, 33 (2018).

${ }^{10}$ T. Fache, H. S. Tarazona, J. Liu, G. L'vova, M. J. Applegate, J. C. Rojas-Sanchez, S. Petit-Watelot, C. V. Landauro, J. Quispe-Marcatoma, R. Morgunov, C. H. W. Barnes, and S. Mangin, Phys. Rev. B 98, 064410 (2018).

${ }^{11}$ S. H. Liang, T. T. Zhang, P. Barate, J. Frougier, M. Vidal, P. Renucci, B. Xu, H. Jaffrès, J. M. George, X. Devaux, M. Hehn, X. Marie, S. Mangin, H. X. Yang, A. Hallal, M. Chshiev, T. Amand, H. F. Liu, D. P. Liu, X. F. Han et al., Phys. Rev. B 90, 085310 (2014).

${ }^{12}$ Y. Luo, M. Moske, and K. Samwer, Europhys. Lett. 42, 565 (1998).

${ }^{13}$ R. Morgunov, G. L'vova, A. Talantsev, O. Koplak, S. Petit-Watelot, X. Devaux, S. Migot, Y. Lu, and S. Mangin, Appl. Phys. Lett. 110, 212403 (2017).

${ }^{14}$ A. I. Veinger, A. G. Zabrodskii, and T. V. Tisnek, Phys. Status Solidi B 230, 107 (2002).

${ }^{15}$ R. Morgunov, M. Farle, M. Passacantando, L. Ottaviano, and O. Kazakova, Phys. Rev. B 78, 045206 (2008).

${ }^{16}$ O. Koplak, A. Talantsev, Y. Lu, A. Hamadeh, P. Pirro, T. Hauet, R. Morgunov, and S. Mangin, J. Magn. Magn. Mater. 433, 91 (2017).

${ }^{17}$ P. Bruno, G. Bayreuther, P. Beauvillain, C. Chappert, G. Lugert, D. Renard, J. P. Renard, and J. Seiden, J. Appl. Phys. 68, 5759 (1990).

${ }^{18} \mathrm{M}$. El-Hilo, A. M. de Witte, K. O’Grady, and R. W. Chantrell, J. Magn. Magn. Mater. 117, L307 (1992).

${ }^{19}$ E. P. Wohlfarth, J. Phys. F: Met. Phys. 14, L155 (1984).

${ }^{20}$ P. Gaunt, Philos. Mag., B 48, 261 (1983).

${ }^{21}$ P. Gaunt, J. Appl. Phys. 59, 4129 (1986).

${ }^{22}$ N. L. Schryer and L. R. Walker, J. Appl. Phys. 45, 5406 (1974).

${ }^{23}$ T. Devolder, P.-H. Ducrot, J.-P. Adam, I. Barisic, N. Vernier, J.-V. Kim, B. Ockert, and D. Ravelosona, Appl. Phys. Lett. 102, 022407 (2013).

${ }^{24}$ Y.-C. Cho, S.-B. Choe, and S.-C. Shin, Appl. Phys. Lett. 80, 452 (2002). 